
\title{
28 Research Square \\ Epidemiology, Clinical Profile and Treatment Outcomes of Bacterial and Fungal Keratitis
}

\author{
Nabila Mabrouk \\ Minia University \\ Mohamed Abdelkader \\ Minia University Faculty of Medicine \\ Mohamed Abdelhakeem \\ Minia University Faculty of Medicine \\ Khaled Mourad \\ Minia University Faculty of Medicine \\ Ahmed Abdelghany ( $\nabla$ ahmeda.abdelghany82@gmail.com ) \\ Minia University Faculty of Medicine https://orcid.org/0000-0003-4632-2315
}

\section{Research Article}

Keywords: Fungal keratitis, Bacterial keratitis, Corneal trauma

Posted Date: June 10th, 2021

DOI: https://doi.org/10.21203/rs.3.rs-573494/v1

License: (c) (i) This work is licensed under a Creative Commons Attribution 4.0 International License. Read Full License

Version of Record: A version of this preprint was published at International Ophthalmology on November 28th, 2021. See the published version at https://doi.org/10.1007/s10792-021-02128-x. 


\section{Abstract}

\section{Purpose:}

The purpose of the study is to determine the microbiological aetiology, epidemiological factors, and clinical profile and treatment outcomes of infective keratitis in Ophthalmology department, Minia University. Egypt

\section{Methods:}

Prospective, non-randomized, observational clinical series of cases, including 150 patients with mean age 30 (range 12 to 85 years), 90 patients (60\%) were males and 60 (40\%) were females, clinically diagnosed as infective corneal ulcer, attending the Ophthalmology Department - Faculty of Medicine. Minia University, Minia, Egypt. From 2018 to 2020.

Detailed history taking and all clinical findings were collected. Corneal scrapings were obtained from patients and subjected to staining and culture for bacterial and fungal pathogens; Bacterial and fungal growth were identified by standard laboratory procedures.

\section{Results:}

Corneal trauma by a vegetative matter was the commesnest risk factor associated with infective keratitis in 92 cases (61.3\%). Smear and Culture was positive in 83 cases $(58.4 \%)$ of 142 corneal scrapings obtained, of which 60 cases were fungal (72.3\%), 21 cases were bacterial (25.3\%) and 2 cases were mixed bacterial and fungal (2.4\%), Aspergillus species was the commonest fungal species isolated in fungal keratitis. 142 cases $(94.67 \%$ ) healed completely with scar. Only 6 cases (4\%) required evisceration due to aggressive presentation from the start and keratoplasty was performed for 2 cases $(1.33 \%)$.

\section{Conclusions:}

Fungal keratitis was the commonest type in cases attending to our department. Adequate diagnosis, management and follow up helped in achieving high successful curative outcomes.

Clinical Trials.gov ID: NCT04894630. Time of registration 1 December 2018

\section{Introduction}

Infective Keratitis is a serious condition that requires immediate and appropriate management to ensure the best anatomical and visual outcome for the patient. [1]

Corneal infections are the leading cause of monocular blindness in some developing countries. Despite the availability of a wide range of newer antimicrobials and new diagnostic techniques, infective corneal ulcer continues to pose a diagnostic and therapeutic challenge. [2] 
The identification of the causative organism is important for the selection of the appropriate type of antimicrobial agent and to avoid the unnecessary use of ineffective and potentially toxic drugs. [3]

Epidemiological and microbiological profile of corneal ulceration has been found to vary with patient population, health of cornea, geographical location and climate. [4]

In this study, we aim to report the epidemiology, the predisposing factors, microbiological profile and the outcome of management of bacterial and fungal corneal ulcers in patients presenting to ophthalmology outpatient clinic at faculty of Medicine, Minia University, Egypt.

\section{Patients And Methods}

This study was a prospective, consecutive nonrandomized clinical series of cases approved by the ethics committee of the Faculty of Medicine, Minia University, Minia, Egypt.

The study is registered in Clinical Trials.gov with ID: NCT04894630.

The study was accepted as electronic poster in European society of cataract and refractive surgeons (ESCRS) Amsterdam. October 2020.

Written informed consent forms were collected from each subject at the time of their first study visit. This study adhered to the principles outlined in the Declaration of Helsinki.

The study included 150 patients with mean age 30 (range 12 to 80 years), 90 patients (60\%) were males and 60 (40\%) were females, clinically diagnosed as infective corneal ulcer, attending the Ophthalmology Department - Faculty of Medicine - Minia University, Minia, Egypt from October 2018 to December 2020.

Detailed history taking and all clinical findings were collected.

Clinical findings included evaluation of visual acuity using snellen chart, and evaluation of the corneal ulcer using slit lamp biomicroscopy to assess site, size, shape, depth and extent of infiltrate. Associated findings like corneal neovascularization; keratic precipitates (KPs), anterior chamber reaction were also recorded.

Corneal ulcer size was graded to either small to medium less than $5 \mathrm{~mm}$ in diameter or large more than 5 $\mathrm{mm}$ in diameter. And depth was graded to superficial (not reaching mid stroma, intermediate (reaching mid stroma) and deep (deeper than mid stroma).

A total of 142 corneal scrapings were obtained from patients. Corneal scrapings were performed under sterile aseptic conditions by the same ophthalmologist (A. A) and processed for smear and culture in appropriate media using standard laboratory protocols. For patients under treatment, antimicrobial treatment was stopped for 48 hours before obtaining specimen for laboratory work up. During this period the patient received conservative nonspecific treatment in the form of: analgesics, topical antiglaucomatous eye drops, and lubricant drops. 
Corneal Scrapings were done under the slit-lamp biomicroscope with the aid of topical anaesthetic eye drops (Benoxinate Hydrochloride $0.4 \%$ ), one or two or more drops were installed then using surgical disposable Baird-Parker blade No.15. Scraping of the ulcerative area was performed in a series of short, moderately firm strokes to sample both the leading edges and the base of each infiltrated area. Scraping was done in one direction from the peripheral margins towards the center of the corneal ulcer.

The material obtained from scrapings and impression was partly smeared on glass slides, subjected to $10 \% \mathrm{KOH}$ (potassium hydroxide) wet mount, Gram and Giemsa stains, and then examined using light microscope for the presence of microorganism.

Another part of the material obtained from scrapings was inoculated in brain heart infusion broth which was incubated at $37^{\circ} \mathrm{C}$, then subcultured on:

1. Blood agar: 2 agar plates were inoculated; one plate incubated aerobically at $37^{\circ} \mathrm{C}$ for aerobic bacteria and another plate incubated in $10 \% \mathrm{CO}_{2}$ enriched jar for anaerobic bacteria and fungi.

2. MacConkey's agar, incubated at $37^{\circ} \mathrm{C}$ for gram negative bacilli.

3. Sabouraud's dextrose agar, incubated at $37^{\circ} \mathrm{C}$ (for fungi).

Primary direct culture was examined after 24 and 48 hours. Subculture was performed from the Brain Heart infusion broth after 48 and 72 hours. All isolated colonies were subjected to full microbiological identification procedures.

Initial growth in Sabouraud's dextrose agar occurs within 72 hours in most cases. But, if no growth after 72 hours, cultures were kept for 1-4 weeks for confirmation of no growth.

\section{Sensitivity Testing:}

Antibiotic as well as antifungal sensitivity were done for the isolated bacterial and/or fungal strains. Antibiotic susceptibility testing was performed, using Bauer-Kirby method in which standardized antibiotic disks were applied on a standardized inoculum of the organism plated as a lawn on the standard Mueller-Hinton medium. After overnight incubation, the inhibition zones around the disks were measured and compared to standard charts according to the type of the microorganism. According to the diameters of the inhibition zones, organisms were determined as sensitive, intermediate or resistant.

Treatment: Patients received medical treatment in the form of topical eye drops according to clinical diagnosis and culture results.

- For fungal keratitis: Natamycin $5 \%$ and Fluconazole topically, and moxifloxacin $0.5 \%$ eye drops for secondary bacterial infection. Systemic antifungal in the form of Itraconazole, $200 \mathrm{mg} /$ day was added for deep ulcers.

For bacterial keratitis: Monotherapy in the form of moxifloxacin $0.5 \%$ eye drops. And modification of therapy according to culture results; Staphylococcus aureus (gram positive bacteria) was highly sensitive 
to vancomycin. Pseudomonas aeruginosa (gram negative bacteria) was sensitive to ceftazidime. Systemic antibacterial treatment (Augmentin; Amoxicillin and Clavulinic acid) was added for deep ulcers.

In addition to cycloplegic eye drops and intraocular pressure lowering agents in cases with corneal thinning. Surgical debridement was performed for diffuse fungal keratitis for better penetration of topical drugs. Keratoplasty was performed for 2 cases and evisceration was performed for 6 cases which have severe corneal melting and total endophthalmitis from the start.

Follow up: Initially twice per week until clinical improvement, then once per week till complete resolution.

\section{Results}

The study included 150 eyes of 150 patients, mean age 30 (12-85 years) with most of patients (73 cases, $48.7 \%$ ), were more than 60 years. 90 cases (60\%) were males and 60 cases (40\%) were females.

144 cases $(96 \%)$ were from rural areas. Farmers represented 77 cases $(51.3 \%)$ followed by 55 cases were housewives (33.3\%). The rest of cases were workers and students (15.4\%).

Trauma by plant origin was the commonest risk factor reported in our study in 92 cases (61.3\%), and only 4 cases had a history of wearing contact lenses and 7 cases had allergic keratoconjuctivitis with topical steroid use. 19 cases had a history of previous eye surgery either refractive corneal surgery or intraocular surgery like cataract surgery or retinal detachment surgery. The rest of cases no local risk factor was defined.

Systemic risk factor was reported in only 9 cases; 8 cases had diabetes mellitus and 1 case had Rheumatoid arthritis.

Corneal ulcer size was less than $5 \mathrm{~mm}$ in diameter in 80 cases (53.3\%) and more than $5 \mathrm{~mm}$ in diameter in 70 cases $(46.7 \%)$.

89 cases $(59.3 \%)$ had superficial ulcers, 55 cases (36.7\%) had corneal ulcer with intermediate depth and 6 cases $(4 \%)$ had deep ulcers.

67 cases $(44.7 \%)$ had anterior chamber reaction in the form of hypopyon.

Corneal scraping was not done in 8 cases as the cornea was very thin. Microbiological investigation of samples of the 142 eyes showed positive Scraping smear in 83 cases (58.45\%), and negative scraping smear in 59 cases $(41.55 \%)$.

Gram stain was positive for gram positive cocci in 14 cases and gram negative bacilli in 9 cases, $\mathrm{KOH}$ preparation and gram stain were positive for hyphae in the same 62 cases.

Microbiological investigation of samples of 142 eyes showed positive culture results in 83 cases (58.4 $\%)$. 
Pure bacterial growth was isolated from 21 cases, pure fungal growth was isolated from 60 cases (Fig. 1), and mixed bacterial and fungal growth was found in two cases.

Staphylococcus aureus was the commonest bacteria isolated from 12 cases followed by Pseudomonas aeruginosa which was isolated from 9 cases followed by streptococcus non hemolyticus which was isolated from 2 cases.

Aspergillus flavus was the commonest fungus and was isolated from 24 cases (Fig. 2) followed by Aspergillus niger which was isolated from 15 cases, followed by aspergillus fumigateus (Fig. 3) which was isolated from 14 cases and Candida albicans which was also isolated from 7 cases.

Mixed growth of Aspergillus niger + staphylococcus aureus was found in two cases.

After receiving the appropriate treatment for each case according to clinical and microbiological results, the success in outcome of treatment was defined as improvement in signs and symptoms and complete healing of keratitis by scarring, which was achieved in 142 cases (94.67\%). Only 6 cases (4\%) required evisceration due to aggressive presentation from the start and keratoplasty was performed for 2 cases (1.33\%). Healing time was about $4-8$ weeks in bacterial keratitis and 6-12 weeks in fungal keratitis.

\section{Discussion}

Clinical experience and careful slit lamp examination could point toward an etiological diagnosis of infective keratitis but Methods for rapid detection of microbial agents and confirmation of clinical diagnosis are very important adequate management of this serious condition like culture and direct microscopic smear examinations of the corneal scrapes. [4]

In the present study, 150 samples from eyes with infective corneal ulcer were studied. All of patients were subjected to detailed history taking, clinical ophthalmological examination, and microbiological laboratory investigation.

In our study, The most susceptible persons to infective corneal ulcers were old age $>60$ years in $48.7 \%$ of cases, not In accordance with Srinivasan et al who found that the most susceptible persons to infective corneal ulcers were adult of $31-60$ years (59\% of 434 cases studied) due to outdoor activity of this age group more than old age in their countries. [5]

The susceptible gender also varies according community. In our study, males were more commonly affected with the disease than females ( $60 \%$ versus $40 \%$ of 150 cases investigated), the ratio being 3:2. This was comparable with the study conducted by Bharathi et al who reported $65.1 \%$ cases to be males and $34.9 \%$ to be females. [6] And in another study male to female ratio was 1:1 [7]

Due to the nature of our government, Most of the patients were from rural areas (96\%). Farmers represented 77 cases (51.3\%) followed by housewives 55 cases (33.3\%). The rest of cases were workers and students (15.4\%). Not In accordance with our results, Sedhu et al who reported that housewives are 
more affected $(21 \%)$ than farmers $(16.9 \%)$, this could be referred to the higher incidence of contact lens wearers in their communities. [8]

Trauma by plant origin was the commonest risk factor reported in in 92 cases (61.3\%), and only 4 cases had a history of wearing contact lenses and 7 cases had allergic keratoconjuctivitis with topical steroid use. 19 cases had a history of previous eye surgery. And the risk factor was not defined in the rest of cases.

This was in agreement with Basak et al who stated that the majority of the cases in their study gave a history of trauma (88\%). [9]

On the other hand, Bourcier et al reported that contact lenses were the main risk factor $(50.3 \%$ in cases of infective corneal ulcers). [10]

Additionally, there was a statistically significant relationship between trauma and the type of the isolated organism ( $p$ value $=0.008$ ). Ocular trauma was the risk factor in all cases of positive fungal culture. This was in agreement with Maung et al who reported that the ocular trauma in the agricultural society was the main cause of fungal ulcer. [11].

Corneal ulcer size was less than $5 \mathrm{~mm}$ in diameter in 80 cases (53.3\%) This was in agreement with Bharathi et al who reported that $66.6 \%$ of cases in their study presented with small corneal ulcers. [1]

Increased depth of the ulcer, larger stromal infiltrates and presence of hypopyon were common in corneal ulcers with positive microbiological studies. This was proven in our results by the statistically significant relation between positive microbiological studies and each of the extent of stromal infiltrates ( $p$-value $=$ 0.001 ) and presence of the hypopyon ( $p$-value $<0.001$ ).

In the present study, when culture results were analyzed, microorganisms were isolated in $58.45 \%$ of the 142 corneal scrapes obtained from microbial infective corneal ulcer. This figure was close to the report of Srinivasan et al were positive culture obtained in $68.4 \%$ of cases [5], but was lower than the reports from Upadhyay et al [12] who reported $80 \%$ and from Dunlop et al who reported $81.7 \%$. [13]

Pure fungal growth was the most prevalent culture result (42.25\%) followed by pure bacterial growth (14.79\%). In $1.41 \%$ of the cases, a mixed growth of bacterial and fungal was found. In $41.5 \%$ of the cases, culture was negative. The fungal ulcer was also predominant in $59 \%$ of cases, than bacterial ulcer in $42 \%$ of cases in a study performed by Basak et al; [9] Etiological pattern of corneal ulceration varies with geographic region, climate and tends to vary over time. [14]

Aspergillus flavus was the commonest fungus and was isolated from 24 cases followed by Aspergillus niger which was isolated from 15 cases, followed by aspergillus fumigateus which was isolated from 14 cases and Candida albicans which was also isolated from 7 cases. 
Staphylococcus aureus was the commonest bacteria isolated from 12 cases followed by Pseudomonas aeruginosa which was isolated from 9 cases followed by streptococcus non hemolyticus which was isolated from 2 cases.

Mixed growth of Aspergillus niger + staphylococcus aureus was found in two cases.

In our study, treatment was started by Empirical topical antimicrobial treatment in addition to adjuvant drugs ( cycloplgics, anti-glaucoma therapy and antibiotics for secondary bacterial infection).

After microbiological examination; if the organism was detected, the antimicrobial was modified according to smear and culture.

Systemic antimicrobial treatment was given only in severe and deep ulcers, but it was not given as a routine, this fact was reported by Upadhyay et al. [15]

In the present study, there was successful treatment outcome with complete healing of keratitis by scarring, which was achieved in 142 cases (94.67\%). Only 6 cases (4\%) required evisceration due to aggressive presentation from the start and keratoplasty was performed for 2 cases (1.33\%) (Fig. 4). Healing time was about 4-8 weeks in bacterial keratitis and 6-12 weeks in fungal keratitis.

In conclusion, the mainstay of management of infective corneal ulcers is early identification of pathogens and proper selection of antimicrobials. Evaluation of epidemiological and microbiological profile of infective keratitis of a region can significantly contributes in adequate and appropriate management of this sight threatening condition.

\section{Declarations}

\section{Funding:}

Not applicable

\section{Conflict of interest:}

None of the authors has any financial disclosure

\section{Availbility of data and material:}

Not applicable

Code availability:

Not applicable

\section{Authors' contributions:}


Nabila A. Mabrouk

Conception and design of study

Data collection

Analysis and interpretation of data

Final approval of the article

Provision of materials

Mohamed F. Osman Abdelkader MD

Conception and design of study

Analysis and interpretation of data

Critical revision of the article

Final approval of the article

Mohamed A. Abdelhakeem MD

Conception and design of study

Analysis and interpretation of data

Critical revision of the article

Final approval of the article

Khaled M. Mourad MD

Conception and design of study

Critical revision of the article

Final approval of the article

\section{Ahmed A. Abdelghany MD}

Conception and design of study

Data collection

Analysis and interpretation of data 
Writing the manuscript

Critical revision of the article

Final approval of the article

Provision of materials

Literature search

\section{Ethical approval:}

The study was approved by the ethics committee of the Faculty of Medicine, Minia University, Minia, Egypt.

\section{Consent to participate:}

Not applicable.

\section{Consent for publication:}

Not applicable now.

\section{References}

1. Bharathi MJ, Ramakrishnan R, Meenakshi R, Mittal S, Shivakumar C, Srinivasan M. Microbiological diagnosis of infective keratitis: comparative evaluation of direct microscopy and culture results. Br J Ophthalmol. 2006; 90(10):1271-1277.

2. Thomas PA, kaliamurthy J, Geraldine P. Epidemiological and Microbiological Diagnosis of Suppurative Keratitis in Gangetic West Bengal, Eastern India. India J Opthalmol. 2005; 53:143.

3. Daniell M, Mills R, Morlet N. Microbial Keratitis: What's the preferred Initial therapy? Br J Ophthalmol. 2003;87:1167-1236.

4. Saurabh Patel, et al. Epidemiologial and microbiological profile of patients having microbial keratitis. National Journal of Community Medicine. 2014; 5: 463-467.

5. Srinivasan M, Gonzales CA, George C, Cevallos V, Mascarenhas JM, Asokan B, et al. Epidemiology and aetiological diagnosis of corneal ulceration in Madurai, South India. Br J Ophthalmol1997; 81:965-1036.

6. Bharathi MJ, Ramakrishnan R, Vasu S, Meenakshi R, Palaniappan R. Aetiological diagnosis of microbial keratitis in South India: A study of 1618 cases. Indian J Med Microbiol. 2002;20:19-24.

7. Schaefer F, Bruttin O, Zogrofos L, Guex-Crosier V. Bacterial keratitis: a prospective clinical and microbiological study. Br J Ophthalmol 2001; 85:842-849. 
8. Sedhu, P. A., Sugathan, S., Pushpakaran, A., \& Kurian, C. Bacterial and fungal profile of infectious keratitis: a prospective study. International Journal of Scientific Study.2017; 5(8), 128-132.

9. Basak SK, Basak S, Mohanta A, Bhowmick A. Epidemiological and microbiological diagnosis of suppurative keratitis in Gangetic West Bengal, eastern India. Indian J Ophthalmol 2005; 53:17-22.

10. Bourcier T, Thomas F, Borderie V, Chaumeil C, Laroche L. Bacterial keratitis. Predisposing factors, clinical and mcrobiological review of 300 cases. Br J Ophthalmol 2003; 87(7):834-842.

11. Maung N, Thant CC; Srinivasan M. Upadhyay MP, Priyadarsini B, Mahalakshmi R, et al. Corneal ulceration in south East: a strategy for the prevention of fungal keratitis at the village level in burma. Br J Ophthalmol 2006; 90 (8):968-1038.

12. Upadhyay MP, Karmacharya PC, Koirala S, Tuladhar NR, Bryan LE, Smolin G, et al. Epidemiologic characteristics, predisposing factors, and etiologic diagnosis of corneal ulceration in Nepal. Am J Ophthalmol 1991;111(1):92-101.

13. Dunlop AA, Wright ED, Howlader SA, Nazrul I, Husain R, McClellan K, et al. Suppurative corneal ulceration in Bangladesh: A study of 142 cases examining the microbiological diagnosis, clinical, and epidemiological features of bacterial and fungal keratitis. Aust NZ J Ophthalmol 1994; 22(2):105-115.

14. Panda A, Satpathy G, Nayak N, Kumar S, Kumar A. Demographic pattern, predisposing factors and management of ulcerative keratitis: evaluation of one thousand unilateral cases at a tertiary care centre. Clin Experiment Ophthalmol 2007; 35:44-50.

15. Upadhyay, Madan P., Muthiah Srinivasan, and John P. Whitcher. "Diagnosing and managing microbial keratitis." Community eye health 28.89 (2015): 3.

\section{Figures}

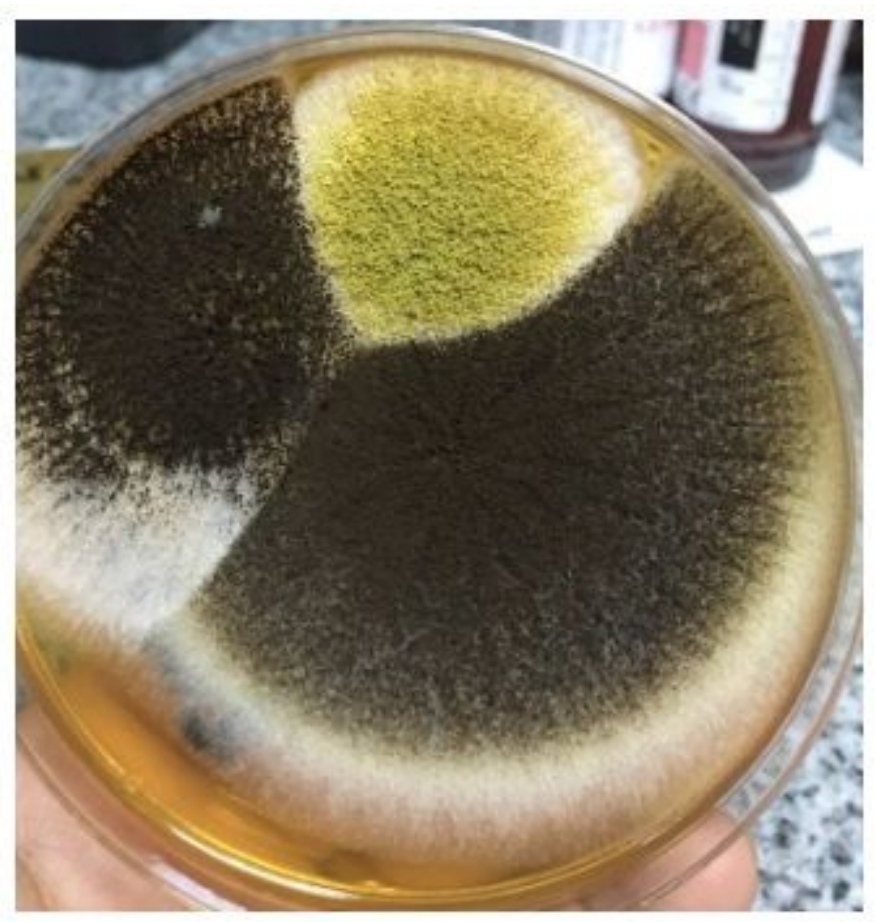




\section{Figure 1}

Aspergillus growth in sabaraud dextrose agar.

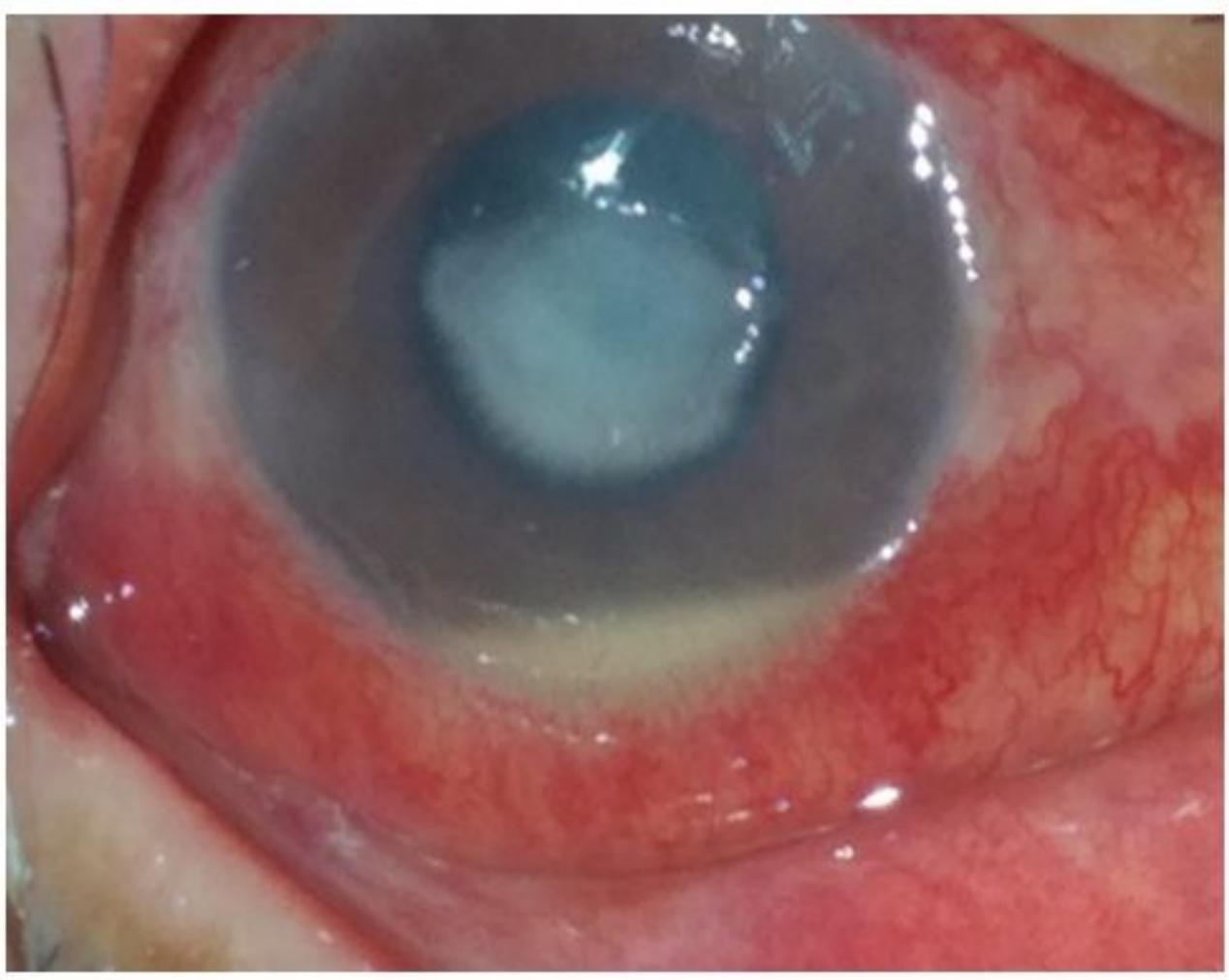

Figure 2

Fungal keratitis caused by aspergillus flavus with hypopyon.

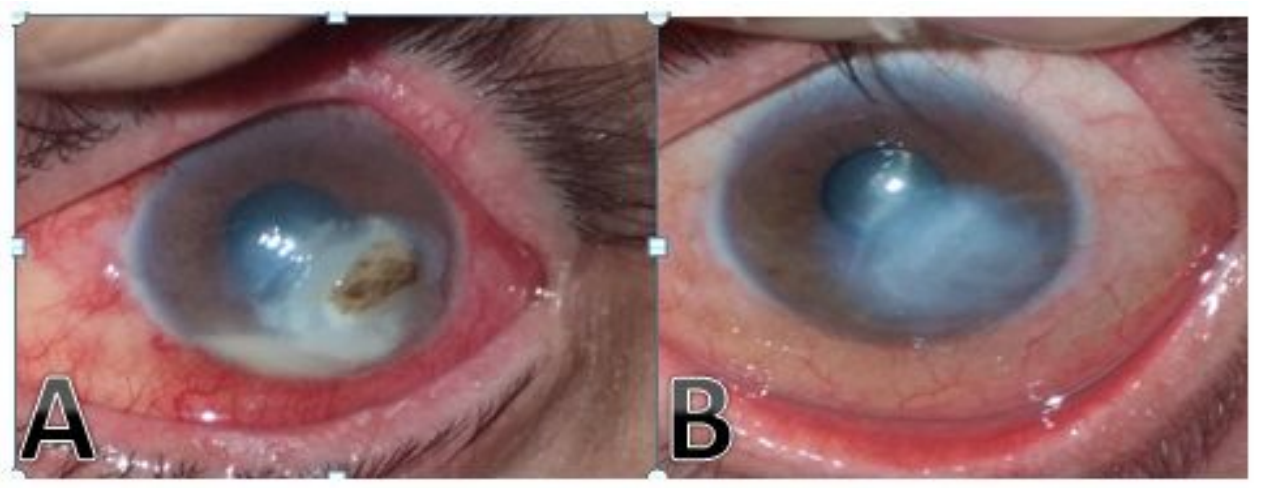

\section{Figure 3}

Fungal keratitis (aspergillus fumigateus) before (A) and after treatment (B). 


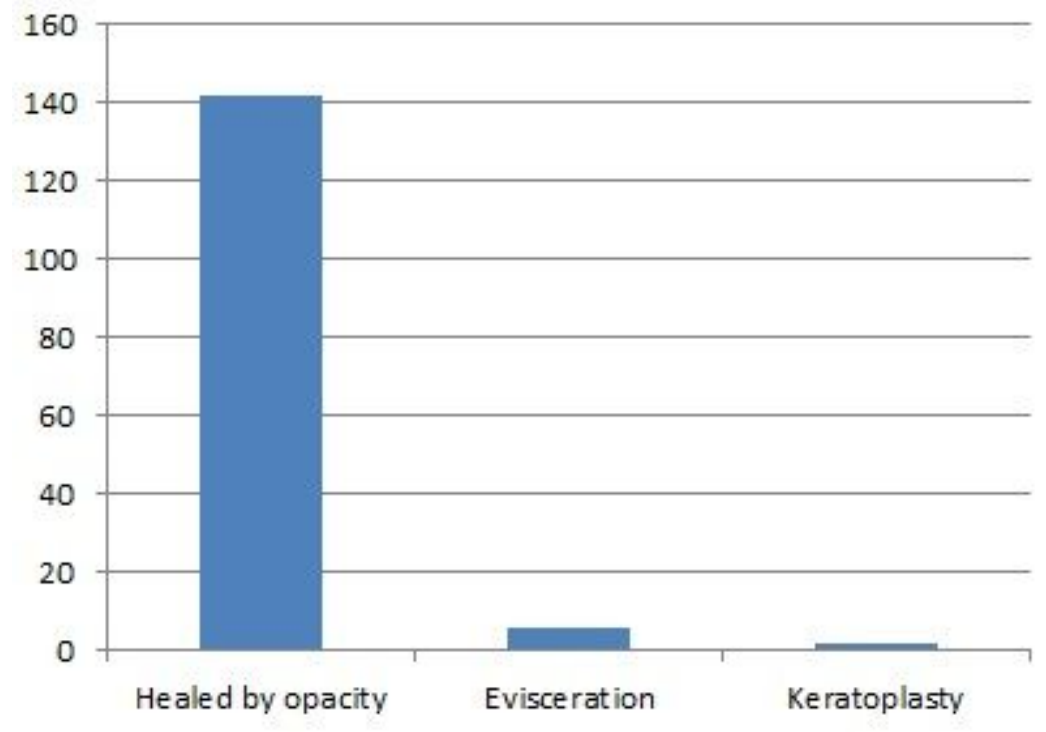

Figure 4

Distribution of the studied cases according to treatment outcomes. 